\title{
Extensions of the natural approach to refinements and generalizations of some trigonometric inequalities
}

\author{
Branko Malešević ${ }^{\text {* }}$ (D), Tatjana Lutovac ${ }^{1}$, Marija Rašajski ${ }^{1}$ and Cristinel Mortici ${ }^{2,3,4}$
}

\section{"Correspondence:}

branko.malesevic@etf.rs

${ }^{1}$ School of Electrical Engineering,

University of Belgrade, Belgrade,

Serbia

Full list of author information is

available at the end of the article

\begin{abstract}
In this paper we propose a new method for sharpening and refinements of some trigonometric inequalities. We apply these ideas to some inequalities of

Wilker-Cusa-Huygens type.
\end{abstract}

MSC: Primary 33B10; secondary 26D05

Keywords: Sharpening; Generalization; Wilker-Cusa-Huygens's inequalities; Sine and cosine functions; Leibniz's theorem for alternating series

\section{Introduction}

Inequalities involving trigonometric functions are used in many applications in various fields of mathematics such as difference equations and inequalities [1], theory of stability, theory of approximations, etc. A method called the natural approach, proposed by Mortici in [2], uses the idea of comparing functions to their corresponding Taylor polynomials. This method has been successfully applied to prove and approximate a wide category of trigonometric inequalities $[3,4]$.

In this paper we extend the ideas of the natural approach by comparing and replacing functions with their corresponding power series. In particular, we focus on the results of Mortici in [2] related to Wilker-Cusa-Huygens's inequalities and give generalizations and refinements of the inequalities stated in Theorems 1, 2, 3, 4, 5, and 6 in that paper. They are cited below.

Statement 1 ([2], Theorem 1) For every $0<x<\pi / 2$, we have

$$
-\frac{x^{4}}{15}<\cos x-\left(\frac{\sin x}{x}\right)^{3}<-\frac{x^{4}}{15}+\frac{23 x^{6}}{1890}
$$

Statement 2 ([2], Theorem 2) For every $0<x<\pi / 2$, we have

$$
-\frac{1}{180} x^{4}<\frac{\sin x}{x}-\frac{\cos x+2}{3}<-\frac{1}{180} x^{4}+\frac{1}{3780} x^{6}
$$

(c) The Author(s) 2018. This article is distributed under the terms of the Creative Commons Attribution 4.0 International License (http://creativecommons.org/licenses/by/4.0/), which permits unrestricted use, distribution, and reproduction in any medium, provided you give appropriate credit to the original author(s) and the source, provide a link to the Creative Commons license, and indicate if changes were made. 
Statement 3 ([2], Theorem 3) For every $0<x<\pi / 2$, we have

$$
3+\left(\frac{3}{20} x^{4}-\frac{3}{140} x^{6}\right) \frac{1}{\cos x}<2 \frac{\sin x}{x}+\frac{\tan x}{x}<3+\frac{3}{20} \frac{x^{4}}{\cos x} .
$$

Statement 4 ([2], Theorem 4) For every $0<x<\pi / 2$, we have

$$
2+\left(\frac{8 x^{4}}{45}-\frac{8 x^{6}}{105}\right) \frac{1}{\cos x}<\left(\frac{\sin x}{x}\right)^{2}+\frac{\tan x}{x}<2+\frac{8 x^{4}}{45} \frac{1}{\cos x} .
$$

Statement 5 ([2], Theorem 5) For every $0<x<\pi / 2$, we have

$$
\left(\frac{x}{\sin x}\right)^{2}+\frac{x}{\tan x}>2+\frac{2}{45} x^{4}
$$

Statement 6 ([2], Theorem 6) For every $0<x<\pi / 2$, we have

$$
3 \frac{x}{\sin x}+\cos x>4+\frac{1}{10} x^{4}+\frac{1}{210} x^{6} .
$$

\section{Preliminaries}

First, let us recall some of the well-known power series expansions that will be used in our proofs.

For $x \in R$, the following power series expansions hold:

$$
\sin x=\sum_{k=0}^{\infty}(-1)^{k} \frac{1}{(2 k+1) !} x^{2 k+1}, \quad \cos x=\sum_{k=0}^{\infty}(-1)^{k} \frac{1}{(2 k) !} x^{2 k}
$$

Also, according to [5], for $x \in R$, we have the following power series expansions:

$$
\cos ^{3} x=\frac{1}{4} \sum_{k=1}^{\infty}(-1)^{k} \frac{3^{2 k}+3}{(2 k) !} x^{2 k}
$$

and

$$
\sin ^{3} x=\frac{1}{4} \sum_{k=1}^{\infty}(-1)^{k+1} \frac{3^{2 k+1}-3}{(2 k+1) !} x^{2 k+1} .
$$

For $x \in\left(0, \frac{\pi}{2}\right)$, according to [5], the following series expansions hold:

$$
\begin{aligned}
& \operatorname{cosec}(x)=\frac{1}{x}+\sum_{k=1}^{\infty} \frac{\left|\boldsymbol{B}_{2 k}\right|\left(2^{2 k}-2\right)}{(2 k) !} x^{2 k-1}, \\
& \operatorname{cosec}^{2}(x)=\frac{1}{x^{2}}+\sum_{k=1}^{\infty} \frac{\left|\boldsymbol{B}_{2 k}\right|(2 k-1) 4^{k}}{(2 k) !} x^{2 k-2},
\end{aligned}
$$

and

$$
\operatorname{cotan}(x)=\frac{1}{x}-\sum_{k=1}^{\infty} \frac{\left|\boldsymbol{B}_{2 k}\right| 4^{k}}{(2 k) !} x^{2 k-1},
$$

where $\boldsymbol{B}_{i}$ are Bernoulli's numbers. 
Theorem WD ([6], Theorem 2) Suppose that $f(x)$ is a real function on $(a, b)$, and that $n$ is a positive integer such that $f^{(k)}(a+), f^{(k)}(b-)(k \in\{0,1,2, \ldots, n\})$ exist.

(i) Supposing that $(-1)^{(n)} f^{(n)}(x)$ is increasing on $(a, b)$, then for all $x \in(a, b)$ the following inequality holds:

$$
\begin{aligned}
& \sum_{k=0}^{n-1} \frac{f^{(k)}(b-)}{k !}(x-b)^{k}+\frac{1}{(a-b)^{n}}\left(f(a+)-\sum_{k=0}^{n-1} \frac{(a-b)^{k} f^{(k)}(b-)}{k !}\right)(x-b)^{n} \\
& \quad<f(x)<\sum_{k=0}^{n} \frac{f^{(k)}(b-)}{k !}(x-b)^{k} .
\end{aligned}
$$

Furthermore, if $(-1)^{n} f^{(n)}(x)$ is decreasing on $(a, b)$, then the reversed inequality of $(7)$ holds.

(ii) Supposing that $f^{(n)}(x)$ is increasing on $(a, b)$, then for all $x \in(a, b)$ the following inequality holds:

$$
\begin{aligned}
& \sum_{k=0}^{n-1} \frac{f^{(k)}(a+)}{k !}(x-a)^{k}+\frac{1}{(b-a)^{n}}\left(f(b-)-\sum_{k=0}^{n-1} \frac{(b-a)^{k} f^{(k)}(a+)}{k !}\right)(x-a)^{n} \\
& >f(x)>\sum_{k=0}^{n} \frac{f^{(k)}(a+)}{k !}(x-a)^{k} .
\end{aligned}
$$

Furthermore, if $f^{(n)}(x)$ is decreasing on $(a, b)$, then the reversed inequality of $(8)$ holds.

Let us mention that an interesting application of Theorem WD is given in [7], see also [8].

\section{Main results}

We need the following theorem for the proofs of Theorems 1, 2, 3, and 4.

Proposition 1 Let the series $f(x)=\sum_{k=1}^{\infty}(-1)^{k+1} A(k) x^{2 k}$ converge for $x \in(0, c), c \in R^{+}$. Suppose that the following statements are true:

(i) If $c<1$, then the sequence $\{A(k)\}_{k \in N}$ is a positive decreasing sequence that converges to 0 .

(ii) If $c \geq 1$, then the sequence $\{A(k)\}_{k \in N}$ is a positive sequence, $\lim _{k \rightarrow+\infty} c^{2 k} A(k)=0$ and $A(k)>c^{2} A(k+1)$ for $k \geq 1$.

Then, for all $x \in(0, c)$ and for all $n \in N$ and $m \in N$, we have

$$
\sum_{k=1}^{2 n}(-1)^{k+1} A(k) x^{2 k}<f(x)<\sum_{k=1}^{2 n+1}(-1)^{k+1} A(k) x^{2 k}
$$

and

$$
\left|f(x)-\sum_{k=1}^{m}(-1)^{k} A(k) x^{2 k}\right|<A(m+1) x^{2 m+2}<c^{2 m+2} A(m+1) .
$$

Proof Suppose that $c<1$. Then, for every $x \in(0, c)$, the positive sequence $\left\{A(k) x^{2 k}\right\}_{k \in N}$ decreases monotonically and $\lim _{k \rightarrow \infty} A(k) x^{2 k}=0$. Thus, assertions (9) and (10) immediately follow from Leibniz's theorem for the alternating series. 
Suppose now that $c \geq 1$. We have

$$
\begin{aligned}
f(x) & =\sum_{k=1}^{\infty}(-1)^{k+1} A(k) x^{2 k} \\
& =\sum_{k=1}^{\infty}(-1)^{k+1} A(k) c^{2 k}\left(\frac{x}{c}\right)^{2 k} .
\end{aligned}
$$

Let us introduce the substitution $t=\frac{x}{c}$ in the previous power series and consider the series

$$
\sum_{k=1}^{\infty}(-1)^{k+1} A(k) c^{2 k} t^{2 k} \quad \text { for } t \in(0,1)
$$

For the assumption $A(k)>c^{2} A(k+1)$, we have

$$
A(k)>c^{2} A(k+1) \quad \Longleftrightarrow A(k) c^{2 k}>c^{2 k+2} A(k+1) \quad \text { for } k \geq 1 .
$$

Hence, we conclude that for every $t \in(0,1)$ power series (11) satisfies Leibniz's theorem for the alternating series, and for all $n, m \in N$ we have

$$
\sum_{k=1}^{2 n}(-1)^{k+1} A(k) c^{2 k} t^{2 k}<\sum_{k=1}^{\infty}(-1)^{k+1} A(k) c^{2 k} t^{2 k}<\sum_{k=1}^{2 n+1}(-1)^{k+1} A(k) c^{2 k} t^{2 k}
$$

and

$$
\begin{aligned}
\left|\sum_{k=1}^{\infty}(-1)^{k+1} A(k) c^{2 k} t^{2 k}-\sum_{k=1}^{m}(-1)^{k+1} A(k) c^{2 k} t^{2 k}\right| & <A(m+1) c^{2 m+2} t^{2 m+2} \\
& <A(m+1) c^{2 m+2}
\end{aligned}
$$

Returning the variable $x=t c$ to (12) and (13) gives the assertions of proposition.

\subsection{Refinements of the inequalities in Statement 1}

We propose the following improvement and generalization of Statement 1.

\section{Theorem 1}

(i) For every $x \in\left(0, \frac{\pi}{2}\right)$ and every $n \in N$, we have

$$
\sum_{k=2}^{2 n}(-1)^{k} A(k) x^{2 k}<\cos x-\left(\frac{\sin x}{x}\right)^{3}<\sum_{k=2}^{2 n+1}(-1)^{k} A(k) x^{2 k},
$$

where

$$
A(k)=\frac{3^{2 k+3}-32 k^{3}-96 k^{2}-88 k-27}{4(2 k+3) !} .
$$

(ii) For every $x \in\left(0, \frac{\pi}{2}\right)$ and every $m \in N$, we have the following error estimation:

$$
\left|\cos x-\left(\frac{\sin x}{x}\right)^{3}-\sum_{k=1}^{m}(-1)^{k} A(k) x^{2 k}\right|<A(m+1) x^{2 m+2} .
$$


Examples Let $x \in\left(0, \frac{\pi}{2}\right)$.

For $n=1$, we get Statement 1 .

For $n>1$, we have the following new results:

- Taking $n=2$ in (14) gives

$$
\begin{aligned}
\frac{1}{15} x^{4}+\frac{23}{1890} x^{6}-\frac{41}{37,800} x^{8} & <\cos x-\left(\frac{\sin x}{x}\right)^{3} \\
& <-\frac{1}{15} x^{4}+\frac{23}{1890} x^{6}-\frac{41}{37,800} x^{8}+\frac{53}{831,600} x^{10}
\end{aligned}
$$

- Taking $n=3$ in (14) gives

$$
\begin{aligned}
& -\frac{1}{15} x^{4}+\frac{23}{1890} x^{6}-\frac{41}{37,800} x^{8}+\frac{53}{831,600} x^{10}-\frac{74,677}{27,243,216,000} x^{12} \\
& <\cos x-\left(\frac{\sin x}{x}\right)^{3} \\
& <-\frac{1}{15} x^{4}+\frac{23}{1890} x^{6}-\frac{41}{37,800} x^{8}+\frac{53}{831,600} x^{10} \\
& \quad-\frac{74,677}{27,243,216,000} x^{12}+\frac{989}{10,897,286,400} x^{14}
\end{aligned}
$$

- Taking $n=4$ in (14) gives

$$
\begin{aligned}
-\frac{x^{4}}{15} & +\frac{23 x^{6}}{1890}-\frac{41 x^{8}}{37,800}+\frac{53 x^{10}}{831,600}-\frac{74,677 x^{12}}{27,243,216,000}+\frac{989 x^{14}}{10,897,286,400} \\
& -\frac{79,649 x^{16}}{33,345,696,384,000} \\
< & \cos x-\left(\frac{\sin x}{x}\right)^{3} \\
< & -\frac{x^{4}}{15}+\frac{23 x^{6}}{1890}-\frac{41 x^{8}}{37,800}+\frac{53 x^{10}}{831,600}-\frac{74,677 x^{12}}{27,243,216,000}+\frac{989 x^{14}}{10,897,286,400} \\
& -\frac{79,649 x^{16}}{33,345,696,384,000}+\frac{454,007 x^{18}}{8,869,955,238,144,000}
\end{aligned}
$$

etc.

Proof of Theorem 1 Consider the function

$$
f(x)=\cos x-\left(\frac{\sin x}{x}\right)^{3} \quad \text { for } x \in\left(0, \frac{\pi}{2}\right)
$$

Based on power series expansions (1) and (3), we have

$$
f(x)=\sum_{k=1}^{\infty}(-1)^{k} A(k) x^{2 k}=\sum_{k=2}^{\infty}(-1)^{k} A(k) x^{2 k}
$$


for all $x \in R$, where

$$
A(k)=\frac{1}{4} \frac{3^{2 k+3}-3}{(2 k+3) !}-\frac{1}{(2 k) !}=\frac{3^{2 k+3}-32 k^{3}-96 k^{2}-88 k-27}{4(2 k+3) !} .
$$

For $c=\pi / 2$, we have

$$
A(k)>0 \quad \text { for } k \geq 2, \quad \text { and } \quad \lim _{k \rightarrow \infty} c^{2 k} A(k)=0 .
$$

Also,

$$
\begin{aligned}
& c^{2} A(k+1)<A(k) \Longleftrightarrow \\
& 32 k^{5}+240 k^{4}+\left(680-8 c^{2}\right) k^{3}+\left(900-45 c^{2}\right) k^{2}+\left(548-\frac{161 c^{2}}{2}\right) k-45 c^{2}+\frac{477}{4} \\
& <\frac{3\left(4 c^{2} k^{2}+18 c^{2} k+20 c^{2}-9\right)}{4} 9^{k+1} .
\end{aligned}
$$

As the last inequality holds for $k \geq 1$, the assertions of Theorem 1 immediately follow from Proposition 1.

\subsection{Refinements of the inequalities in Statement 2}

We propose the following improvement and generalization of Statement 2.

\section{Theorem 2}

(i) For every $x \in\left(0, \frac{\pi}{2}\right)$ and every $n \in N$, we have

$$
\sum_{k=2}^{2 n}(-1)^{k+1} B(k) x^{2 k}<\frac{\sin x}{x}-\frac{\cos x+2}{3}<\sum_{k=2}^{2 n+1}(-1)^{k+1} B(k) x^{2 k},
$$

where

$$
B(k)=\frac{2}{3} \frac{k-1}{(2 k+1) !} .
$$

(ii) For every $x \in\left(0, \frac{\pi}{2}\right)$ and every $m \in N$, we have the following error estimation:

$$
\left|\frac{\sin x}{x}-\frac{\cos x+2}{3}-\sum_{k=0}^{m}(-1)^{k+1} B(k) x^{2 k}\right|<B(m+1) x^{2 m+2} .
$$

Examples Let $x \in\left(0, \frac{\pi}{2}\right)$.

For $n=1$, we get Statement 2 .

For $n>1$, we have the following new results:

- Taking $n=2$ in (17) gives

$$
\begin{aligned}
-\frac{1}{180} x^{4}+\frac{1}{3780} x^{6}-\frac{1}{181,440} x^{8} & <\frac{\sin x}{x}-\frac{1}{3} \cos x-\frac{2}{3} \\
& <-\frac{1}{180} x^{4}+\frac{1}{3780} x^{6}-\frac{1}{181,440} x^{8}+\frac{1}{14,968,800} x^{10} ;
\end{aligned}
$$


- Taking $n=3$ in (17) gives

$$
\begin{aligned}
- & \frac{x^{4}}{180}+\frac{x^{6}}{3780}-\frac{x^{6}}{181,440}+\frac{x^{10}}{14,968,800}-\frac{x^{12}}{1,868,106,240} \\
& <\frac{\sin x}{x}-\frac{1}{3} \cos x-\frac{2}{3} \\
& <-\frac{x^{4}}{180}+\frac{x^{6}}{3780}-\frac{x^{8}}{181,440}+\frac{x^{10}}{14,968,800}-\frac{x^{12}}{1,868,106,240}+\frac{x^{14}}{326,918,592,000}
\end{aligned}
$$

etc.

Proof of Theorem 2 Consider the function

$$
f(x)=\frac{\sin x}{x}-\frac{1}{3} \cos x-\frac{2}{3} \quad \text { for } x \in\left(0, \frac{\pi}{2}\right) .
$$

Based on power series expansion (1), we have

$$
f(x)=-\frac{2}{3}+\sum_{k=0}^{\infty}(-1)^{k+1} \frac{2}{3} \frac{k-1}{(2 k+1) !} x^{2 k}=\sum_{k=2}^{\infty}(-1)^{k+1} \frac{2}{3} \frac{k-1}{(2 k+1) !} x^{2 k} .
$$

The sequence $\{B(k)\}_{k \in N, k \geq 2}$ satisfies the recurrence relation

$$
B(k+1)=\frac{k}{2\left(k^{2}-1\right)(2 k+3)} B(k) .
$$

For $c=\pi / 2$, we have

$$
B(k)>0 \quad \text { for } k \geq 2, \quad \text { and } \quad \lim _{k \rightarrow \infty} c^{2 k} B(k)=0 .
$$

Also,

$$
\begin{aligned}
c^{2} B(k+1)<B(k) & \Longleftrightarrow\left(\frac{c^{2} k}{2\left(k^{2}-1\right)(2 k+3)}-1\right) \cdot B(k)<0 \\
& \Longleftrightarrow-\frac{4 k^{3}+6 k^{2}-\left(c^{2}+4\right) k-6}{2\left(k^{2}-1\right)(2 k+3)} \cdot B(k)<0 \\
& \Longleftrightarrow-\frac{2\left(2 k^{3}-3\right)+4 k(k-1)+k\left(2 k-c^{2}\right)}{2\left(k^{2}-1\right)(2 k+3)} \cdot B(k)<0 .
\end{aligned}
$$

As the last inequality holds for every $k \geq 2$, the assertions of Theorem 2 follow from Proposition 1.

\subsection{Refinements of the inequalities in Statement 3}

We propose the following improvement and generalization of Statement 3.

\section{Theorem 3}

(i) For every $x \in\left(0, \frac{\pi}{2}\right)$ and every $n \in N$, we have

$$
3+\frac{1}{\cos x} \sum_{k=2}^{2 n+1}(-1)^{k} C(k) x^{2 k}<2 \frac{\sin x}{x}+\frac{\tan x}{x}<3+\frac{1}{\cos x} \sum_{k=2}^{2 n}(-1)^{k} C(k) x^{2 k},
$$


where

$$
C(k)=2 \frac{4^{k}-3 k-1}{(2 k+1) !} .
$$

(ii) For every $x \in\left(0, \frac{\pi}{2}\right)$ and every $m \in N, m \geq 2$, we have the following error estimation:

$$
\left|2 \frac{\sin x}{x}+\frac{\tan x}{x}-\left(3+\frac{1}{\cos x} \sum_{k=2}^{m}(-1)^{k+1} C(k) x^{2 k}\right)\right|<C(m+1) \frac{x^{2 m+2}}{\cos x} .
$$

Examples Let $x \in\left(0, \frac{\pi}{2}\right)$.

For $n=1$, we get Statement 3 .

For $n>1$, we have the following new results:

- Taking $n=2$ in (19) gives

$$
\begin{aligned}
2+ & \frac{1}{\cos x}\left(\frac{3}{20} x^{4}-\frac{3}{140} x^{6}+\frac{3}{2240} x^{8}-\frac{1}{19,800} x^{10}\right) \\
& <2 \frac{\sin x}{x}+\frac{\tan x}{x} \\
& <2+\frac{1}{\cos x}\left(\frac{3}{20} x^{4}-\frac{3}{140} x^{6}+\frac{3}{2240} x^{8}\right) ;
\end{aligned}
$$

- Taking $n=3$ in (19) gives

$$
\begin{aligned}
2+ & \frac{1}{\cos x}\left(\frac{3}{20} x^{4}-\frac{3}{140} x^{6}+\frac{3}{2240} x^{8}-\frac{1}{19,800} x^{10}\right. \\
& \left.+\frac{151}{115,315,200} x^{12}-\frac{101}{4,036,032,000} x^{14}\right) \\
< & 2 \frac{\sin x}{x}+\frac{\tan x}{x} \\
< & 2+\frac{1}{\cos x}\left(\frac{3}{20} x^{4}-\frac{3}{140} x^{6}+\frac{3}{2240} x^{8}-\frac{1}{19,800} x^{10}+\frac{151}{115,315,200} x^{12}\right)
\end{aligned}
$$

etc.

Proof of Theorem 3 Consider the function

$$
f(x)=\frac{\sin 2 x}{x}+\frac{\sin x}{x}-3 \cos x
$$

for $x \in\left(0, \frac{\pi}{2}\right)$. Based on power series expansion (1), we have

$$
\begin{aligned}
f(x) & =\sum_{k=0}^{\infty}(-1)^{k}\left(\frac{2^{(2 k+1)}}{(2 k+1) !}+\frac{1}{(2 k+1) !}-\frac{3}{(2 k) !}\right) x^{2 k} \\
& =\sum_{k=2}^{\infty}(-1)^{k} 2 \frac{4^{k}-3 k-1}{(2 k+1) !} x^{2 k}
\end{aligned}
$$


for $x \in\left(0, \frac{\pi}{2}\right)$. For $c=\pi / 2$, we have

$$
C(k)>0 \quad \text { for } k \geq 2, \quad \text { and } \quad \lim _{k \rightarrow \infty} c^{2 k} C(k)=0 .
$$

Also,

$$
\begin{aligned}
& c^{2} C(k+1)<C(k) \Longleftrightarrow \\
& -2\left(12 k^{3}+38 k^{2}+\frac{\left(6 c^{2}+76\right) k}{2}+4 c^{2}+12\right)<4^{k+1}(2(k+c)(k-c)+5 k+3) .
\end{aligned}
$$

As the last inequality holds for $k \geq 2$, the assertions of Theorem 2 immediately follow from Proposition 1.

\subsection{Refinements of the inequalities in Statement 4}

We propose the following improvement and generalization of Statement 4.

\section{Theorem 4}

(i) For every $x \in\left(0, \frac{\pi}{2}\right)$ and every $n \in N$, we have

$$
\begin{aligned}
2+\frac{1}{\cos x} \sum_{k=2}^{2 n+1}(-1)^{k} D(k) x^{2 k} & <\left(\frac{\sin x}{x}\right)^{2}+\frac{\tan x}{x} \\
& <2+\frac{1}{\cos x} \sum_{k=2}^{2 n}(-1)^{k} D(k) x^{2 k},
\end{aligned}
$$

where

$$
D(k)=\frac{1}{4} \frac{-9+3^{2 k+2}-40 k-32 k^{2}}{(2 k+2) !} .
$$

(ii) For every $x \in\left(0, \frac{\pi}{2}\right)$ and every $m \in N, m \geq 2$, we have the following error estimation:

$$
\left|\left(\frac{\sin x}{x}\right)^{2}+\frac{\tan x}{x}-\left(2+\frac{1}{\cos x} \sum_{k=2}^{m}(-1)^{k+1} D(k) x^{2 k}\right)\right|<D(m+1) \frac{x^{2 m+2}}{\cos x} .
$$

Examples Let $x \in\left(0, \frac{\pi}{2}\right)$.

For $n=1$, we get Statement 4 .

For $n>1$, we have the following new results:

- Taking $n=2$ in (20) gives

$$
\begin{aligned}
2+ & \frac{1}{\cos x}\left(\frac{8}{45} x^{4}-\frac{4}{105} x^{6}+\frac{19}{4725} x^{8}-\frac{37}{133,650} x^{10}\right) \\
& <\left(\frac{\sin x}{x}\right)^{2}+\frac{\tan x}{x} \\
& <2+\frac{1}{\cos x}\left(\frac{8}{45} x^{4}-\frac{4}{105} x^{6}+\frac{19}{4725} x^{8}\right) ;
\end{aligned}
$$


- Taking $n=3$ in (20) gives

$$
\begin{aligned}
2+ & \frac{1}{\cos x}\left(\frac{8}{45} x^{4}-\frac{4}{105} x^{6}+\frac{19}{4725} x^{8}-\frac{37}{133,650} x^{10}\right. \\
& \left.+\frac{283}{20,638,800} x^{12}-\frac{3503}{6,810,804,000} x^{14}\right) \\
< & \left(\frac{\sin x}{x}\right)^{2}+\frac{\tan x}{x} \\
< & 2+\frac{1}{\cos x}\left(\frac{8}{45} x^{4}-\frac{4}{105} x^{6}+\frac{19}{4725} x^{8}\right. \\
& \left.-\frac{37}{133,650} x^{10}+\frac{283}{20,638,800} x^{12}\right)
\end{aligned}
$$

etc.

Proof of Theorem 4 Consider the function

$$
f(x)=\frac{\cos x-\cos ^{3} x}{x^{2}}+\frac{\sin x}{x}-2 \cos x \quad \text { for } x \in\left(0, \frac{\pi}{2}\right) .
$$

Based on power series expansion (2), we have

$$
\begin{aligned}
f(x) & =\sum_{k=0}^{\infty}(-1)^{k}\left(-\frac{1}{(2 k+2) !}+\frac{1}{4} \frac{3^{(2 k+2)}+3}{(2 k+2) !}+\frac{1}{(2 k+1) !}-\frac{2}{(2 k) !}\right) x^{2 k} \\
& =\sum_{k=0}^{\infty}(-1)^{k} \frac{9^{k+1}-\left(32 k^{2}+40 k+9\right)}{4(2 k+2) !} x^{2 k} \\
& =\sum_{k=2}^{\infty}(-1)^{k} \frac{9^{k+1}-\left(32 k^{2}+40 k+9\right)}{4(2 k+2) !} x^{2 k} .
\end{aligned}
$$

For $c=\pi / 2$, we have

$$
D(k)>0 \quad \text { for } k \geq 2, \quad \text { and } \quad \lim _{k \rightarrow \infty} c^{2 k} D(k)=0 .
$$

Also,

$$
\begin{aligned}
& c^{2} D(k+1)<D(k) \Longleftrightarrow \\
& 32 k^{4}+152 k^{3}+\left(245-8 c^{2}\right) k^{2}+\left(\frac{303}{2}-26 c^{2}\right) k+27-\frac{81 c^{2}}{4} \\
& \quad<\frac{1}{4} 9^{k+1}\left(4 k^{2}+14 k+12-81 c^{2}\right) .
\end{aligned}
$$

As the last inequality holds for $k \geq 2$, the assertions of Theorem 2 immediately follow from Proposition 1. 


\subsection{Refinements of the inequalities in Statement 5}

We prove the following generalization of Statement 5.

Theorem 5 For every $x \in\left(0, \frac{\pi}{2}\right)$ and $m \in N, m \geq 2$, the following inequalities hold:

$$
\begin{aligned}
2 & +\sum_{k=2}^{m-1} \frac{\left|\boldsymbol{B}_{2 k}\right|(2 k-2) 4^{k}}{(2 k) !} x^{2 k}+\left(\frac{2 x}{\pi}\right)^{2 n}\left(\frac{\pi^{2}}{4}-2-\sum_{k=2}^{m-1} \frac{\left|\boldsymbol{B}_{2 k}\right|(2 k-2) 4^{k}}{(2 k) !}\left(\frac{\pi}{2}\right)^{2 k}\right) \\
> & \left(\frac{x}{\sin x}\right)^{2}+\frac{x}{\tan x} \\
& >2+\sum_{k=2}^{m} \frac{\left|\boldsymbol{B}_{2 k}\right|(2 k-2) 4^{k}}{(2 k) !} x^{2 k},
\end{aligned}
$$

where $\boldsymbol{B}_{i}$ are Bernoulli's numbers.

Proof of Theorem 5 Consider the function

$$
f(x)=\left(\frac{x}{\sin x}\right)^{2}+\frac{x}{\tan x} \quad \text { for } x \in\left(0, \frac{\pi}{2}\right)
$$

Based on series expansion (5) and (6), we have

$$
\begin{aligned}
f(x) & =x^{2}\left(\frac{1}{x^{2}}+\sum_{k=1}^{\infty} \frac{\left|\boldsymbol{B}_{2 k}\right|(2 k-1) 4^{k}}{(2 k) !} x^{2 k-2}\right)+x\left(\frac{1}{x}-\sum_{k=1}^{\infty} \frac{\left|\boldsymbol{B}_{2 k}\right| 4^{k}}{(2 k) !} x^{2 k-1}\right) \\
& =2+\sum_{k=1}^{\infty} \frac{\left|\boldsymbol{B}_{2 k}\right|(2 k-1) 4^{k}-4^{k}}{(2 k) !} x^{2 k} \\
& =2+\sum_{k=2}^{\infty} \frac{\left|\boldsymbol{B}_{2 k}\right|(2 k-2) 4^{k}}{(2 k) !} x^{2 k} \\
& >2+\sum_{k=2}^{m} \frac{\left|\boldsymbol{B}_{2 k}\right|(2 k-2) 4^{k}}{(2 k) !} x^{2 k} .
\end{aligned}
$$

Since all coefficients are positive, by applying Theorem WD, we get the inequalities in the statement of the theorem.

Examples For $x \in\left(0, \frac{\pi}{2}\right)$ and $f(x)=\left(\frac{x}{\sin x}\right)^{2}+\frac{x}{\tan x}$, we show the inequalities for $m=2,3,4,5$.

- For $m=2$ :

$$
2+\left(\frac{2}{\pi}\right)^{4}\left(-2+\frac{\pi^{2}}{4}\right) x^{4}>f(x)>2+\frac{2}{45} x^{4}
$$

On the right-hand side we see the inequality from Statement 5.

- For $m=3$ :

$$
2+\frac{2}{45} x^{4}+\left(\frac{2}{\pi}\right)^{6}\left(-2+\frac{\pi^{2}}{4}-\frac{\pi^{4}}{360}\right) x^{6}>f(x)>2+\frac{2}{45} x^{4}+\frac{8}{945} x^{6} .
$$


- For $m=4$ :

$$
\begin{aligned}
2+ & \frac{2}{45} x^{4}+\frac{8}{945} x^{6}+\left(\frac{2}{\pi}\right)^{8}\left(-2+\frac{\pi^{2}}{4}-\frac{\pi^{4}}{360}-\frac{\pi^{6}}{7560}\right) x^{8} \\
> & f(x)>2+\frac{2}{45} x^{4}+\frac{8}{945} x^{6}+\frac{2}{1575} x^{8}
\end{aligned}
$$

- For $m=5$ :

$$
\begin{aligned}
2+ & \frac{2}{45} x^{4}+\frac{8}{945} x^{6}+\frac{2}{1575} x^{8}+\left(\frac{2}{\pi}\right)^{10}\left(-2+\frac{\pi^{2}}{4}-\frac{\pi^{4}}{360}-\frac{\pi^{6}}{7560}-\frac{\pi^{8}}{201,600}\right) x^{10} \\
> & f(x)>2+\frac{2}{45} x^{4}+\frac{8}{945} x^{6}+\frac{2}{1575} x^{8}+\frac{16}{93,555} x^{10} .
\end{aligned}
$$

Remark Let us notice that Theorem WD allows for the approximation error to be estimated. The difference between the right-hand side and the left-hand side of the double inequality in Theorem 5 can be represented by the following function:

$$
R_{n}(x)=\left(f\left(\frac{\pi}{2}\right)-2-\sum_{k=1}^{n} \frac{\left|\boldsymbol{B}_{2 k}\right|(2 k-2) 4^{k}}{(2 k) !}\left(\frac{\pi}{2}\right)^{2 k}\right)\left(\frac{2 x}{\pi}\right)^{2 n} .
$$

The maximum values of $R_{n}(x)$ are reached at $\frac{\pi}{2}$, and their values for $n=3,4,5$ and 6 are $6.97 \times 10^{-2}, 2.26 \times 10^{-2}, 6.95 \times 10^{-3}$, and $2.06 \times 10^{-3}$, respectively.

\subsection{Refinements of the inequalities in Statement 6}

We propose the following generalization of Statement 6.

Theorem 6 For every $x \in\left(0, \frac{\pi}{2}\right)$ and $m \in N, m \geq 3$, the following inequality holds:

$$
\begin{aligned}
4 & +\sum_{k=1}^{m-1} \frac{3\left|\boldsymbol{B}_{2 k}\right|\left(2^{2 k}-2\right)+(-1)^{k}}{(2 k) !} x^{2 k} \\
& +\left(\frac{2 x}{\pi}\right)^{2 m}\left(f\left(\frac{\pi}{2}\right)-4-\sum_{k=1}^{m-1} \frac{3\left|\boldsymbol{B}_{2 k}\right|\left(2^{2 k}-2\right)+(-1)^{k}}{(2 k) !}\left(\frac{\pi}{2}\right)^{2 k}\right) \\
> & 3 \frac{x}{\sin x}+\cos x>4+\sum_{k=1}^{m} \frac{3\left|\boldsymbol{B}_{2 k}\right|\left(2^{2 k}-2\right)+(-1)^{k}}{(2 k) !} x^{2 k},
\end{aligned}
$$

where $\boldsymbol{B}_{i}$ are Bernoulli's numbers.

Proof of Theorem 6 Consider the function

$$
f(x)=3 \frac{x}{\sin x}+\cos x \quad \text { for } x \in\left(0, \frac{\pi}{2}\right) .
$$

Based on the series expansion (1) and (4), we have

$$
f(x)=4+\sum_{k=1}^{\infty} \frac{3\left|\boldsymbol{B}_{2 k}\right|\left(2^{2 k}-2\right)+(-1)^{k}}{(2 k) !} x^{2 k}
$$


It is easy to verify that $3\left|\boldsymbol{B}_{2 k}\right|\left(2^{2 k}-2\right)>1$ for $k \geq 2$, and that it is equal to 1 for $k=1$, therefore all corresponding coefficients are positive. Now, using Theorem WD, we get the inequalities in the statement of the theorem.

Examples For $x \in\left(0, \frac{\pi}{2}\right)$ and $f(x)=3 \frac{x}{\sin x}+\cos x$, we show the inequalities for $m=3,4,5,6$.

- For $m=3$ :

$$
4+\frac{1}{10} x^{4}+\left(\frac{2}{\pi}\right)^{6}\left(-4+\frac{3 \pi}{2}-\frac{\pi^{4}}{160}\right) x^{6}>f(x)>4+\frac{1}{10} x^{4}+\frac{1}{210} x^{6} .
$$

On the right-hand side we see the inequality from Statement 6 .

- For $m=4$ :

$$
\begin{gathered}
4+\frac{1}{10} x^{4}+\frac{1}{210} x^{6}+\left(\frac{2}{\pi}\right)^{8}\left(-4+\frac{3 \pi}{2}-\frac{\pi^{4}}{160}-\frac{\pi^{6}}{13,440}\right) x^{8} \\
>f(x)>4+\frac{1}{10} x^{4}+\frac{1}{210} x^{6}+\frac{11}{16,800} x^{8} .
\end{gathered}
$$

- For $m=5$ :

$$
\begin{aligned}
4+ & \frac{1}{10} x^{4}+\frac{1}{210} x^{6}+\frac{11}{16,800} x^{8} \\
& +\left(\frac{2}{\pi}\right)^{10}\left(-4+\frac{3 \pi}{2}-\frac{\pi^{4}}{160}-\frac{\pi^{6}}{13,440}-\frac{11 \pi^{8}}{4,300,800}\right) x^{10} \\
> & f(x)>4+\frac{1}{10} x^{4}+\frac{1}{210} x^{6}+\frac{11}{16,800} x^{8}+\frac{53}{831,600} x^{10} .
\end{aligned}
$$

- For $m=6$ :

$$
\begin{aligned}
& 4+\frac{x^{4}}{10}+\frac{x^{6}}{210}+\frac{11 x^{8}}{16,800}+\frac{53 x^{10}}{831,600} \\
& \quad+\left(\frac{2}{\pi}\right)^{12}\left(-4+\frac{3 \pi}{2}-\frac{\pi^{4}}{160}-\frac{\pi^{6}}{13,440}-\frac{11 \pi^{8}}{4,300,800}-\frac{53 \pi^{10}}{851,558,400}\right) x^{12} \\
& >f(x)>4+\frac{x^{4}}{10}+\frac{x^{6}}{210}+\frac{11 x^{8}}{16,800}+\frac{53 x^{10}}{831,600}+\frac{117,911 x^{12}}{18,162,144,000} .
\end{aligned}
$$

Remark The difference between the right-hand side and the left-hand side of the double inequality in Theorem 6 can be represented by the following function:

$$
R_{n}(x)=\left(f\left(\frac{\pi}{2}\right)-4+\sum_{k=1}^{n} \frac{3\left|\boldsymbol{B}_{2 k}\right|\left(2^{2 k}-2\right)+(-1)^{k}}{(2 k) !}\left(\frac{\pi}{2}\right)^{2 k}\right)\left(\frac{2 x}{\pi}\right)^{2 n} .
$$

The maximum values of $R_{n}(x)$ are reached at $\frac{\pi}{2}$, and their values for $n=3,4,5$, and 6 are $3.20 \times 10^{-2}, 7.78 \times 10^{-3}, 1.95 \times 10^{-3}$, and $4.88 \times 10^{-4}$, respectively.

\section{Conclusion}

The idea to compare and replace functions with their corresponding power series to get more accurate approximations was used in [9, 10], and [7]. Following the same idea, in 
this paper we extended the natural approach. We proposed and proved new inequalities which represent refinements and generalizations of the inequalities stated in [2], related to Wilker-Cusa-Huygens's inequalities.

Note that proofs of the new inequalities (14), (17), (19), (20), (21), and (22) for any fixed $n, m \in N$ can be obtained by substituting $x=\sin t$ for $t \in\left[0, \frac{\pi}{2}\right]$ and using the methods and algorithms developed in [11] and [12]. However, our approach provides proofs for the approximation of the corresponding function by the inequality of an arbitrary degree.

The results of the present research can be used to verify as well as to refine a broad category of inequalities. For example, Bercu ([13], Theorem 2.4) proved the following inequalities:

$$
\begin{aligned}
& \left(\frac{x}{\sin x}\right)^{2}+\frac{x}{\tan x} \\
& \quad>\frac{11,220 x^{10}-205,560 x^{8}-14,256,000 x^{6}+512,179,200 x^{4}-3,157,056,000 x^{2}+13,716,864,000}{242 x^{12}-8580 x^{10}+25,560 x^{8}-1,080,000 x^{6}+103,680,000 x^{4}-1,578,528,000 x^{2}+6,858,432,000} \\
& \quad>2+\frac{2}{45} x^{4}>2
\end{aligned}
$$

for every $x \in(0, b)$, where $b=\sqrt{\frac{240-6 \sqrt{1090}}{17}}=1.5701 \ldots<\frac{\pi}{2}$.

According to Theorem 5, taking $m=7$ in (21) gives

$$
\left(\frac{x}{\sin x}\right)^{2}+\frac{x}{\tan x}>P_{7}(x) \quad \text { for } x \in\left(0, \frac{\pi}{2}\right)
$$

where

$$
P_{7}(x)=2+\sum_{k=2}^{7} \frac{\left|\boldsymbol{B}_{2 k}\right|(2 k-2) 4^{k}}{(2 k) !} x^{2 k} .
$$

Thus Bercu's inequality is reduced to the following decidable problem (see $[14,15])$ : for every $x \in\left(0, \frac{\pi}{2}\right)$, it is true that

$$
\begin{aligned}
& P_{7}(x) \\
& \qquad>\frac{11,220 x^{10}-205,560, x^{8}-14,256,000 x^{6}+512,179,200 x^{4}-3,157,056,000 x^{2}+13,716,864,000}{242 x^{12}-8580 x^{10}+25,560 x^{8}-1,080,000 x^{6}+103,680,000 x^{4}-1,578,528,000 x^{2}+6,858,432,000} .
\end{aligned}
$$

The above inequality is a refinement of the inequality obtained by Bercu. Moreover, the above inequality shows that Bercu's inequality holds true over an extended interval $\left(0, \frac{\pi}{2}\right)$. Similarly, Theorems 1, 2, 3, 4, 5, and 6 can be applied to other results and inequalities from $[13,16]$, as well as to a broad category of analytical inequalities.

\section{Acknowledgements}

The research of the first, second, and third authors was supported in part by the Serbian Ministry of Education, Science and Technological Development, under Projects ON 174032 \& III 44006, TR 32023, and ON 174033, respectively.

\section{Competing interests}

The authors would like to state that they do not have any competing interests in the subject of this research.

\section{Authors' contributions}

All of the authors participated in every phase of the research conducted for this paper. All authors read and approved the final manuscript. 


\section{Author details}

'School of Electrical Engineering, University of Belgrade, Belgrade, Serbia. ${ }^{2}$ Valahia University of Târgovişte, Târgovişte, Romania. ${ }^{3}$ Academy of Romanian Scientists, Bucharest, Romania. ${ }^{4}$ University Politehnica of Bucharest, Bucharest, Romania.

\section{Publisher's Note}

Springer Nature remains neutral with regard to jurisdictional claims in published maps and institutional affiliations.

Received: 27 December 2017 Accepted: 5 March 2018 Published online: 14 March 2018

\section{References}

1. Agarwal, R.P.: Difference Equations and Inequalities: Theory, Methods, and Applications. Dekker, New York (1992)

2. Mortici, C.: The natural approach of Wilker-Cusa-Huygens inequalities. Math. Inequal. Appl. 14(3), 535-541 (2011)

3. Anderson, D.G., Vuorinen, M., Zhang, X.: Topics in special functions III. In: Milovanović, G.V., Rassias, T.M. (eds.) Analytic Number Theory, Approximation Theory and Special Functions, pp. 297-345. Springer, Berlin (2014)

4. Mitrinović, D.S.: Analytic Inequalities. Springer, Berlin (1970)

5. Gradshteyn, I., Ryzhik, I.: Table of Integrals Series and Products, 8th edn. Academic Press, San Diego (2015)

6. Wu, S., Debnath, L.: A generalization of L'Hospital-type rules for monotonicity and its application. Appl. Math. Lett. 22, 284-290 (2009)

7. Malešević, B., Rašajski, M., Lutovac, T.: A new approach to the sharpening and generalizations of Shafer-Fink and Wilker type inequalities. arXiv:1712.03772

8. Makragić, M.: A method for proving some inequalities on mixed hyperbolic-trigonometric polynomial functions. J. Math. Inequal. 11(3), 817-829 (2017)

9. Malešević, B., Rašajski, M., Lutovac, T.: Refinements and generalizations of some inequalities of Shafer-Fink's type for the inverse sine function. J. Inequal. Appl. 2017, 275 (2017)

10. Malešević, B., Rašajski, M., Lutovac, T.: Refined estimates and generalizations of inequalities related to the arctangent function and Shafer's inequality. arXiv:1711.03786

11. Malešević, B., Makragić, M.: A method for proving some inequalities on mixed trigonometric polynomial functions. J. Math. Inequal. 10(3), 849-876 (2016)

12. Lutovac, T., Malešević, B., Mortici, C.: The natural algorithmic approach of mixed trigonometric-polynomial problems. J. Inequal. Appl. 2017, 116 (2017)

13. Bercu, G.: The natural approach of trigonometric inequalities_Padé approximant. J. Math. Inequal. 11(1), 181-191 (2017)

14. Tarski, A.: A Decision Method for Elementary Algebra and Geometry. University of California Press, Berkeley (1951)

15. Poonen, B.: Undecidable problems: a sampler. In: Kennedy, J. (ed.) Interpreting Gödel: Critical Essays. Chapter 10, pp. 211-241. Cambridge University Press, Cambridge (2014). http://www-math.mit.edu/ poonen/papers/sampler. pdf

16. Bercu, G.: Padé approximant related to remarkable inequalities involving trigonometric functions. J. Inequal. Appl. 2016, 99 (2016)

\section{Submit your manuscript to a SpringerOpen ${ }^{\circ}$ journal and benefit from:}

- Convenient online submission

- Rigorous peer review

Open access: articles freely available online

- High visibility within the field

Retaining the copyright to your article

Submit your next manuscript at $\gg$ springeropen.com 